\title{
A systematic review and meta-analysis on the effect of angiogenesis blockade for the treatment of gastric cancer
}

This article was published in the following Dove Press journal:

OncoTargets and Therapy

\section{Zhi-Gang Bai \\ Zhong-Tao Zhang}

Department of General Surgery, Beijing Friendship Hospital, Capital Medical University, Beijing Key Laboratory of Cancer Invasion and Metastasis Research and National Clinical Research Center for Digestive Diseases, Beijing 100050, People's Republic of China
Correspondence: Zhong-Tao Zhang Department of General Surgery, Beijing Friendship Hospital, Capital Medical University, Beijing Key Laboratory of Cancer Invasion and Metastasis Research and National Clinical Research Center for Digestive Diseases, 95 Yong-An Road, Xi-Cheng District, Beijing 100050, People's Republic of China $\mathrm{Tel} / \mathrm{fax}+861063138745$

Emailx8s8aa@I63.com
Introduction: To date, anti-vascular endothelial growth factor (VEGF) monoclonal antibody ( $\mathrm{mAb}$, bevacizumab), anti-VEGF receptor $\mathrm{mAb}$ (ramucirumab) and selective vascular endothelial growth factor receptor (VEGFR) tyrosine kinase inhibitors (sunitinib, sorafenib and apatinib) have been tested in the clinical trials.

Materials and methods: In the current study, results of 32 clinical trials (24 Phase I or II, 8 Phase III) were systematically reviewed and meta-analysis was performed in 8 Phase III trial results. Results: It was found that median overall survival (OS) time and progression-free survival (PFS) time were significantly longer in the patients treated with antiangiogenic reagents compared to that in the patients with placebo when all of 8 Phase III clinical trials were analyzed together $(\mathrm{OS}$ : odds ratio $=0.805,95 \% \mathrm{CI}: 0.719-0.901, P<0.001$; PFS: odds ratio $=0.719$, 95\% CI: $0.533-969, P=0.030)$.

Conclusion: Meta-analysis on bevacizumab (4 out 8 Phase III trials) indicated that neither OS nor PFS was significantly different between the groups treated with bevacizumab or placebo with or without combination of other chemotherapeutic reagents (OS: odds ratio $=0.909,95 \% \mathrm{CI}$ : $0.780-1.059, P=0.221$;FS: odds ratio $=0.985,95 \%$ CI: $0.865-1.122, P=0.826)$. By contrast, meta-analysis on ramucirumab ( 3 out of 8 Phase III trials) revealed that ramucirumab was significantly favored in the treatment of gastric cancer with significant different OS between the two groups (odds ratio $=0.720,95 \% \mathrm{CI}: 0.604-0.858, P<0.001$ ). In addition, patients treated with VEGF or VEGFR blockers had higher morbidity of hypertension and neutropenia, but lower risk of side effects of vomiting and anemia. These findings suggest that addition of antiangiogenesis reagents, especially anti-VEGFR-mAb, to the first- or second-line chemotherapy could prolong patient's OS and PFS time in the advanced or metastatic gastric cancer.

Keywords: anti-VEGF monoclonal antibody, anti-VEGF receptor mAb, VEGFR tyrosine kinase inhibitors, Phase III trial, overall survival, progression-free survival, chemotherapy

\section{Introduction}

Despite improvements in systemic chemotherapy, the prognosis of advanced or metastatic gastric cancer remains poor. Recent progress in understanding the molecular biology of gastric cancer and the related signaling pathways provides promising strategies for the targeted therapies for the treatment of gastric cancer. In this context, angiogenesis is widely considered as one of the main processes for tumor progression. Vascular endothelial growth factor (VEGF), also known as VEGF-A, is the primary driver of this angiogenesis process in the solid tumors. The family of VEGF molecules also includes VEGF-B, VEGF-C, VEGF-D, VEGF-E and placental growth factor. Each component of this family can bind to several VEGF receptors (VEGFR), known as 
VEGFR1, VEGFR2 and VEGFR3, and 2 co-receptors, neuropilin 1 and 2. In particular VEGF-A binds to VEGFR-1 and -2 , and the most important receptor is VEGFR-2, ${ }^{1-3}$ which regulates the proliferation of endothelial cells through a number of different mechanisms. ${ }^{1,4}$

Although there are no validated biomarkers to select patients for antiangiogenic therapy, strategies of targeting angiogenic process in solid tumors include 1) targeting pro-angiogenic factors (VEGF) with monoclonal antibody $(\mathrm{mAb}) ; 2)$ targeting angiogenic receptors (VEGFR) with $\mathrm{mAb}$ and 3 ) selectively targeting VEGFR associated tyrosine kinase with inhibitors (TKI). To date, two humanized mAbs, bevacizumab (anti-VEGF-mAb) and ramucirumab (antiVEGFR-mAb), have been approved and tested in the varying phases of clinical trials. In addition, VEGFR-selective TKIs, such as sorafenib, sunitinib and apatinib, have also been tested in clinical trials for the treatment of common solid tumors including gastric cancer.

Results of clinical trials with the aforementioned VEGF blockers including anti-VEGF-mAb, anti-VEGFR-mAb and VEGFR-TKIs, however, were inconsistent. The current study was, therefore, designed to systematically review results of the clinical trials for the treatment of gastric cancer with antiVEGF-mAb, anti-VEGFR-mAb or VEGFR-TKIs, and furthermore to perform meta-analysis on the Phase III clinical trials.

\section{Materials and methods}

\section{Data sources}

Relevant literature up to October 10th, 2017, was searched in the sites of PubMed, Embase and Web of Science with the following phrases: "gastric cancer" and "VEGF, VEGFR antagonist" or "VEGF, VEGFR blockade" or "VEGF, VEGFR inhibitor" or "VEGFR tyrosine kinase inhibitor" and "Clinical trial". The search was limited to English and Chinese. In addition, relevant literatures were also identified by hand-searching the references.

\section{Inclusion and exclusion criteria}

Following studies were included into the current systematic review and meta-analysis: 1) clinical studies on the treatment of primary or metastatic gastric cancer with anti-VEGF-mAb (bevacizumab), anti-VEGFR-mAb (ramucirumab) inhibitors or VEGFR-TKIs (sorafenib, sunitinib, apatinib) with or without combination of other chemotherapeutic reagents and 2) studies with full text articles. Studies were excluded if they were preclinical studies or published in a language other than English.

\section{Data extraction}

Data extraction was conducted by two investigators (ZB and ZZ). Data extraction included study name (the first author' last name), year of publication, treatment regimen, total number of cases for each treatment group, median overall survival (OS) months, median progression-free survival (PFS) month and morbidity of adverse effects (Table 1). The senior author (ZB) was involved in consulting for the eligibility of a study if a divergence between the two dataextracting investigators existed.

\section{Statistical analysis}

The following format of data entry was used: 1) median survival month of OS, number of cases and $P$-value; 2 ) median survival month of disease or progression-free interval, number of cases and $P$-value and 3 ) computerized odds ratio and $P$-value. The strength of therapeutic effect by VEGFR blockers on gastric cancer was measured by odds ratio, and the morbidity of side effect was measured by risk ratio. A fixed effect model was applied when no heterogeneity was observed among the studies. Alternatively, a random effect model was applied if the heterogeneity between studies was $P<0.10$ and $I^{2}>50 \%$, which was considered as heterogeneous between the studies. ${ }^{5,6}$ All meta-analysis was performed using the Comprehensive Meta-analysis software (Version 3, NJ, USA).

Table I Summary of data extraction of Phase III clinical trials for meta-analysis

\begin{tabular}{lllll}
\hline Year & Author & Country & Treatment & Cancer type \\
\hline 2011 & Ohtsu et al $^{8}$ & Multi-countries & Bev + Cis + Cap & Advanced gastric cancer \\
2014 & Fuchs et al $^{16}$ & Multi-countries & Ram after first-line & Advanced gastric/gastroesophageal \\
2014 & Wilke et al $^{17}$ & Multi-countries & Ram + Pac & Advanced gastric/gastroesophageal \\
2015 & Ma et al ${ }^{18}$ & China & Bev + Doc/Oxa/5-FU & Locally advanced gastric cancer \\
2015 & Shen et al ${ }^{19}$ & China & Bev + Cisp/Cap & Advanced or metastatic gastric/gastroesophageal \\
2016 & ${\text { Al-Batran et al }{ }^{20}}_{2013}$ & Multi-countries & Ram + Pac & Advanced gastric/gastroesophageal \\
2017 & Li et al $^{36}$ & China & Apatinib & Advanced or metastatic gastric/gastroesophageal \\
\hline
\end{tabular}

Abbreviations: Bev, bevacizumab; Cis, cisplatin; Cap, capecitabine; Doc, docetaxel; Oxa, oxaliplatin; Ram, ramucirumab; Pac, paclitaxel. 


\section{Results}

\section{General information of the enrolled studies}

As shown in Figure 1, after careful reading of the abstracts, 43 full-text articles were retrieved. The articles were then independently assessed, and data were extracted by two investigators. After excluding reviews and case report articles, 32 articles were included in the systematic review ${ }^{7-36}$ and 8 articles of Phase III clinical trials were further included in the meta-analysis. ${ }^{8,16-20,22,23,37}$ Of the 32 articles for systematic review and meta-analysis, 6 articles were from the USA; ${ }^{7,10,13,28,29,38} 5$ articles were from Korea; ${ }^{24,26,30,35} 4$ articles were from multiple centers in different countries; $8,16,17,20$ 5 articles were from People's Republic of China; ${ }^{18,19,21,22,36}$ 4 were from Japan, ${ }^{9,11,25,34} 2$ were from Germany; ${ }^{27,32}$ one each was from Israel, ${ }^{12}$ Netherlands, ${ }^{14}$ Spain $^{31}$ and UK. ${ }^{23}$

Commonly used agents for targeting VEGF/VEGFR signaling pathways were bevacizumab, ramucirumab, apatinib, sunitinib and sorafenib. Of them, bevacizumab is a mAb that targets VEGF-A, which was studied in 4 out of 8 Phase III clinical trials, ${ }^{8,18,19,23}$ ramucirumab is a mAb that targets VEGF receptor, which was studied in 3 out of 8 Phase III clinical trials. ${ }^{16,17,20}$ One out 8 Phase III clinical trials was on the effect of apatinib, a selective VEGFR TKIs. ${ }^{22}$

\section{Effect of targeting VEGF or VEGFR on OS and PFS}

Of the 32 articles selected for systematic review, 8 studies were randomized Phase III trials on the effect of anti-VEGF$\mathrm{mAb}$ (bevacizumab), anti-VEGFR-mAb (ramucirumab) or selective VEGFR-TKI inhibitors (apatinib) in comparison with placebo in combination with or without chemotherapy for the treatment of gastric cancers, ${ }^{8,16-20,22,23,37}$ and 24 studies were Phase I or II trials on effect and safety of anti-VEGF-mAb, anti-VEGFR-mAb or VEGFR-TKI inhibitors. ${ }^{7-15,21,24-36}$

By quantitative meta-analysis of the selected 8 Phase III trials, it was found that median OS time was significantly

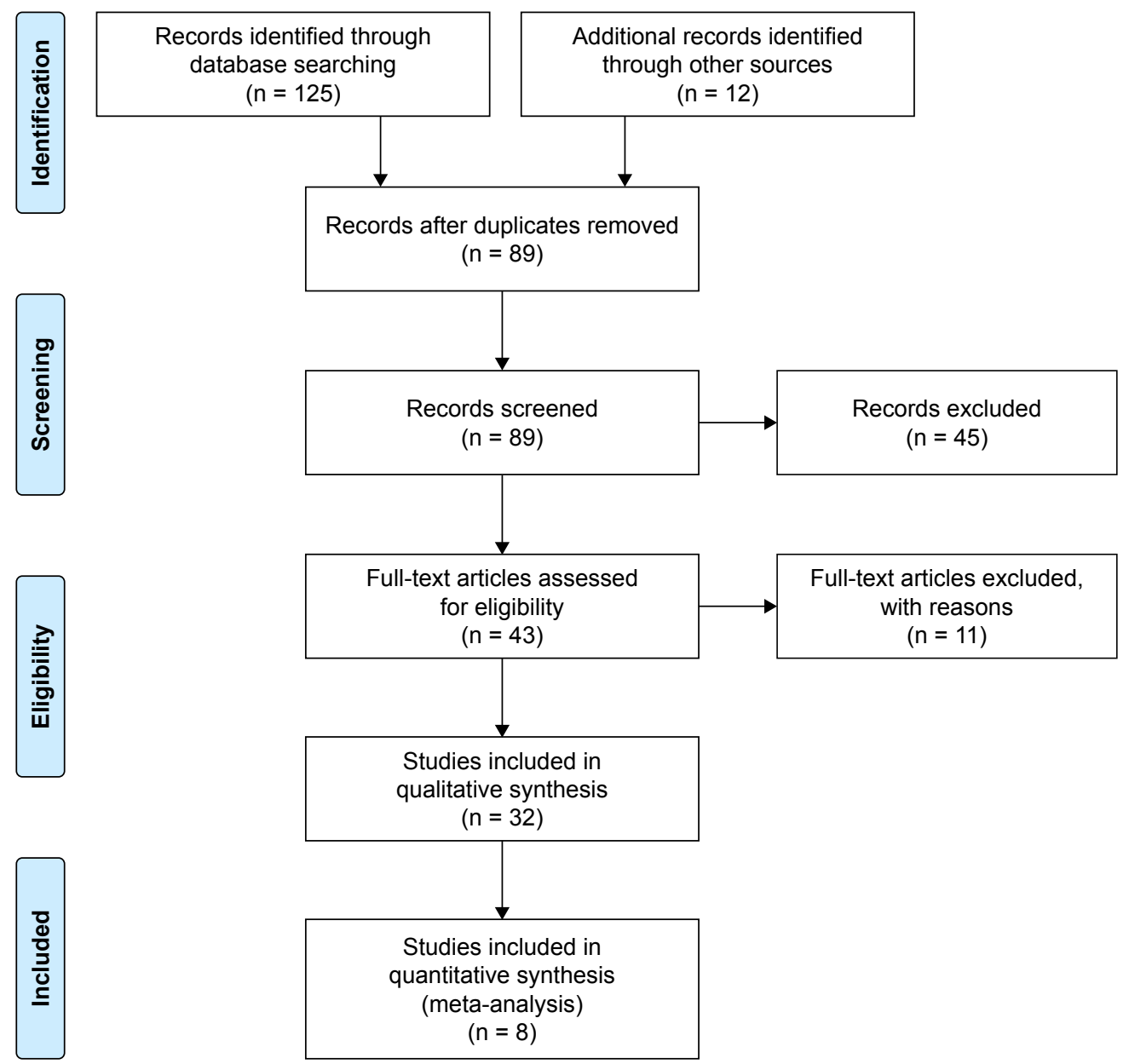

Figure I Flow chart of database search and literature selection. 


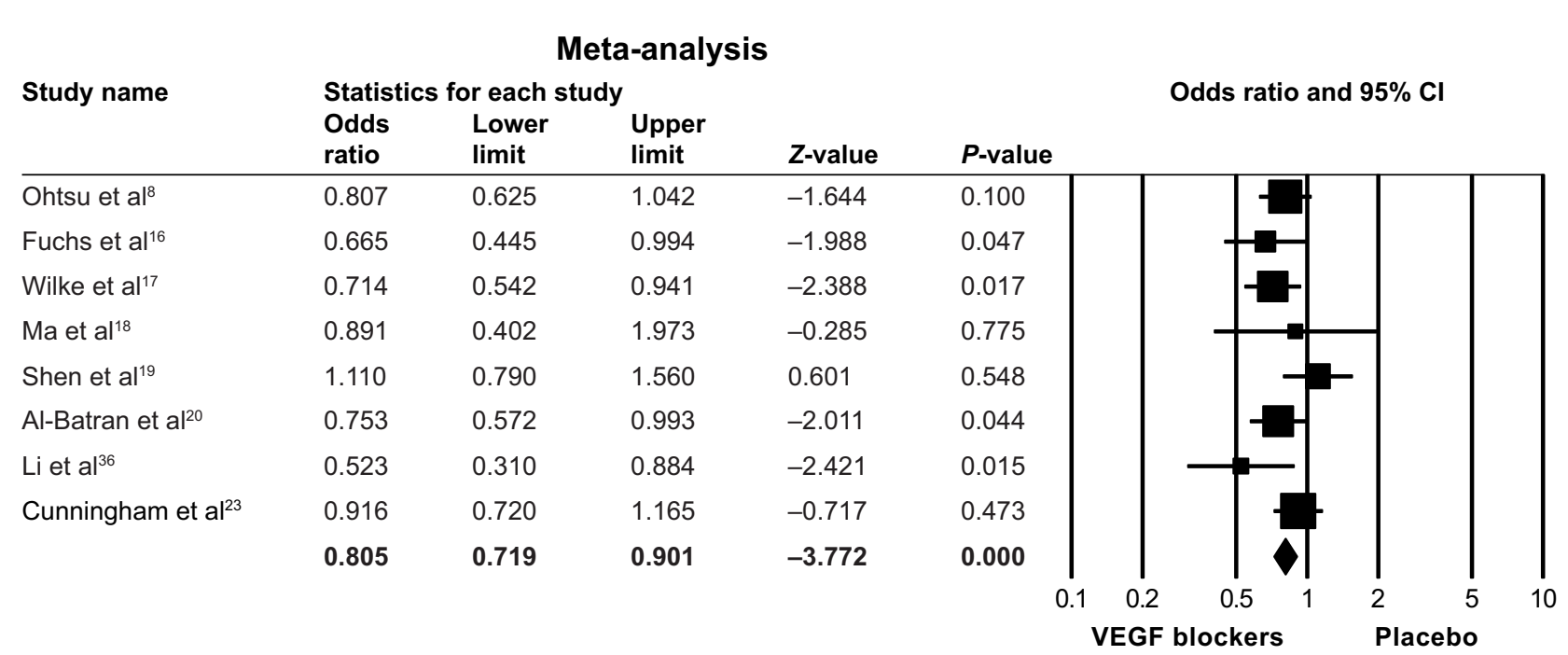

Figure 2 Forest plot for median survival time of gastric cancer patients with or without vascular endothelial growth factor (VEGF) or vascular endothelial growth factor receptor (VEGFR) blocker in addition to chemotherapy.

Notes: A fixed effect model was used due to non-significant heterogeneity of publications $\left(I^{2}=22.17, P=0.253\right)$. Effect size was assessed by odds ratio and $95 \% \mathrm{Cl}$, and the median overall survival (OS) time was in favor VEGF or VEGFR blocker therapy (odds ratio $=0.805 ; 95 \% \mathrm{Cl}$ : 0.7 I $9-0.90$ I, $P<0.00 \mathrm{I}$ ). Bold values represent the median.

longer in the patients treated with VEGF/VEGFR blockers compared to that in the patients without the blockers (odds ratio $=0.805,95 \% \mathrm{CI}: 0.719-0.901, P<0.001$, Figure 2$)$, although Ohtsu et $\mathrm{al}^{8}{ }^{8}$ Shen et $\mathrm{al}^{19}$ and Cunningham et $\mathrm{al}^{23}$ reported that there was no significant difference in overall median survival time between the patients treated with bevacizumab or placebo. Furthermore, as shown in Figure $3 \mathrm{~A}$ and $\mathrm{B}$, when meta-analysis was performed on the results of
A

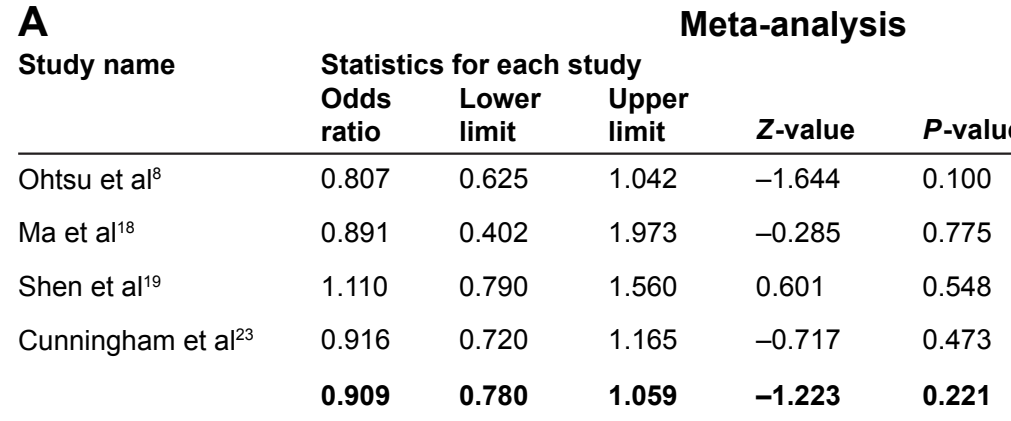

Odds ratio and $95 \% \mathrm{Cl}$

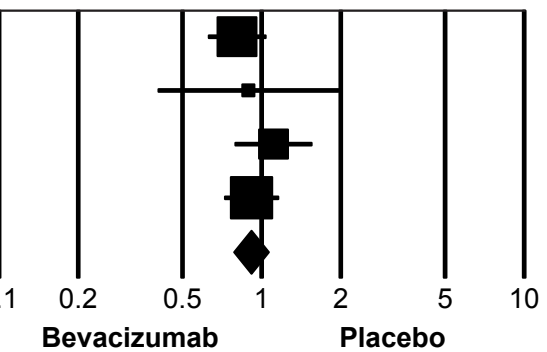

B Study name

\begin{tabular}{|c|c|c|c|c|c|}
\hline \multirow[t]{2}{*}{ Study name } & \multicolumn{5}{|c|}{ Statistics for each study } \\
\hline & $\begin{array}{l}\text { Odds } \\
\text { ratio }\end{array}$ & $\begin{array}{l}\text { Lower } \\
\text { limit }\end{array}$ & $\begin{array}{l}\text { Upper } \\
\text { limit }\end{array}$ & Z-value & $P$-value \\
\hline Fuchs et $\mathrm{al}^{16}$ & 0.665 & 0.445 & 0.994 & -1.988 & 0.047 \\
\hline Wilke et $\mathrm{al}^{17}$ & 0.714 & 0.542 & 0.941 & -2.388 & 0.017 \\
\hline \multirow[t]{2}{*}{ Al-Batran et $\mathrm{al}^{20}$} & 0.753 & 0.572 & 0.993 & -2.011 & 0.044 \\
\hline & 0.720 & 0.604 & 0.858 & -3.666 & 0.000 \\
\hline
\end{tabular}

Odds ratio and $95 \% \mathrm{Cl}$

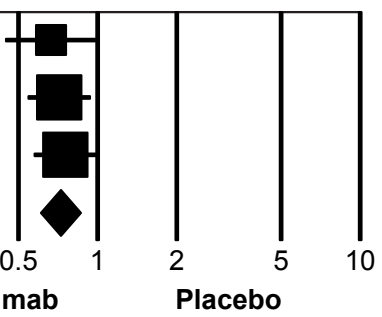

Figure 3 Forest plot for overall survival (OS) for patients with anti-vascular endothelial growth factor (VEGF)-mAb (bevacizumab) or anti-vascular endothelial growth factor receptor (VEGFR)-mAb (ramucirumab) in addition to chemotherapy.

Notes: (A) OS with bevacizumab. A fixed effect model was used due to significant heterogeneity of publications $\left(P^{2}=0, P=0.539\right)$. Effect size was assessed by odds ratio and $95 \% \mathrm{Cl}$, and the OS time was not favored with bevacizumab in addition to chemotherapy (odds ratio $=0.909,95 \% \mathrm{Cl}: 0.780-1.053, P=0.22 \mathrm{I}$ ). (B) OS with ramucirumab. A fixed effect model was used due to significant heterogeneity of publications $\left(I^{2}=0, P=0.880\right)$. Effect size was assessed by odds ratio and $95 \% \mathrm{Cl}$, and the $\mathrm{OS}$ time was not favored with bevacizumab in addition to chemotherapy (odds ratio $=0.720,95 \% \mathrm{Cl}: 0.604-0.858, P<0.00 \mathrm{I}$ ). Bold values represent the median. 


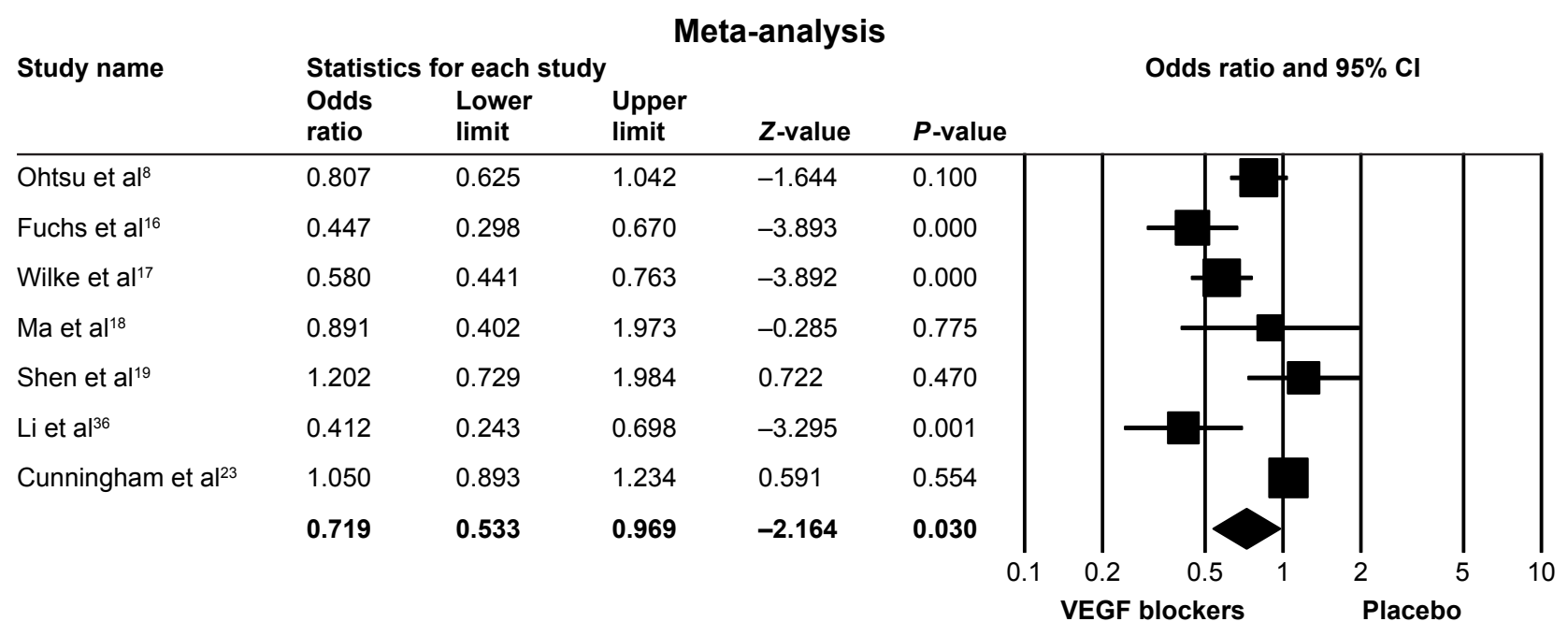

Figure 4 Forest plot for progression-free survival (PFS) for patients with or without vascular endothelial growth factor (VEGF) or vascular endothelial growth factor receptor (VEGFR) blocker in addition to chemotherapy.

Notes: A random effect model was used due to significant heterogeneity of publications $\left(I^{2}=81.56, P<0.0 \mathrm{I}\right)$. Effect size was assessed by odds ratio and $95 \% \mathrm{Cl}$, and the $\mathrm{PFS}$ time was in favor of VEGF or VEGFR blocker in addition to chemotherapy (odds ratio $=0.821,95 \% \mathrm{Cl}$ : $0.735-0.917, P<0.00 \mathrm{I}$ ). Bold values represent the median.

ramucirumab (3 out of 8 Phase III trials) or bevacizumab ( 4 out of 8 Phase III trials), ramucirumab treatment was favored in terms of OS (odds ratio $=0.720,95 \% \mathrm{CI}: 0.604-0.858$, $P<0.001$, Figure 3B), while bevacizumab was not favored in comparison to the placebo treatment $(\mathrm{OS}$ : odds ratio $=$ 0.909, 95\% CI: 0.780-1.059, $P=0.221$, Figure 3A).

VEGF/VEGFR blockers were favored in terms of PFS time. The PFS was significantly longer in patients treated with VEGF/VEGFR blockers than that in patients treated with placebo (odds ratio $=0.719,95 \%$ CI: $0.533-0.969$, $P=0.030$, Figure 4). Furthermore, Phase I or II studies indicated that average PFS of gastric cancer patients treated with VEGF/VEGFR blockers in combination with commonly used chemotherapeutic drugs such as oxaliplatin or cisplatin and docetaxel was 10.5 months (6.6-15.1). ${ }^{7-15}$ All of the 24 Phase I/II clinical studies without proper controls demonstrated that humanized mAbs of targeting angiogenesis were safe to use although various side effects were inevitable.

\section{Side effects of VEGF/VEGFR blockers}

Most of the 32 articles enrolled into the current systematic review and meta-analysis reported that various adverse effects were associated with VEGF/VEGFR blockers. Most common side effects of using VEGF/VEGFR blockers were hypertension, vomiting, neutropenia and anemia. As shown in Figure 5 of the meta-analysis result, patients treated with

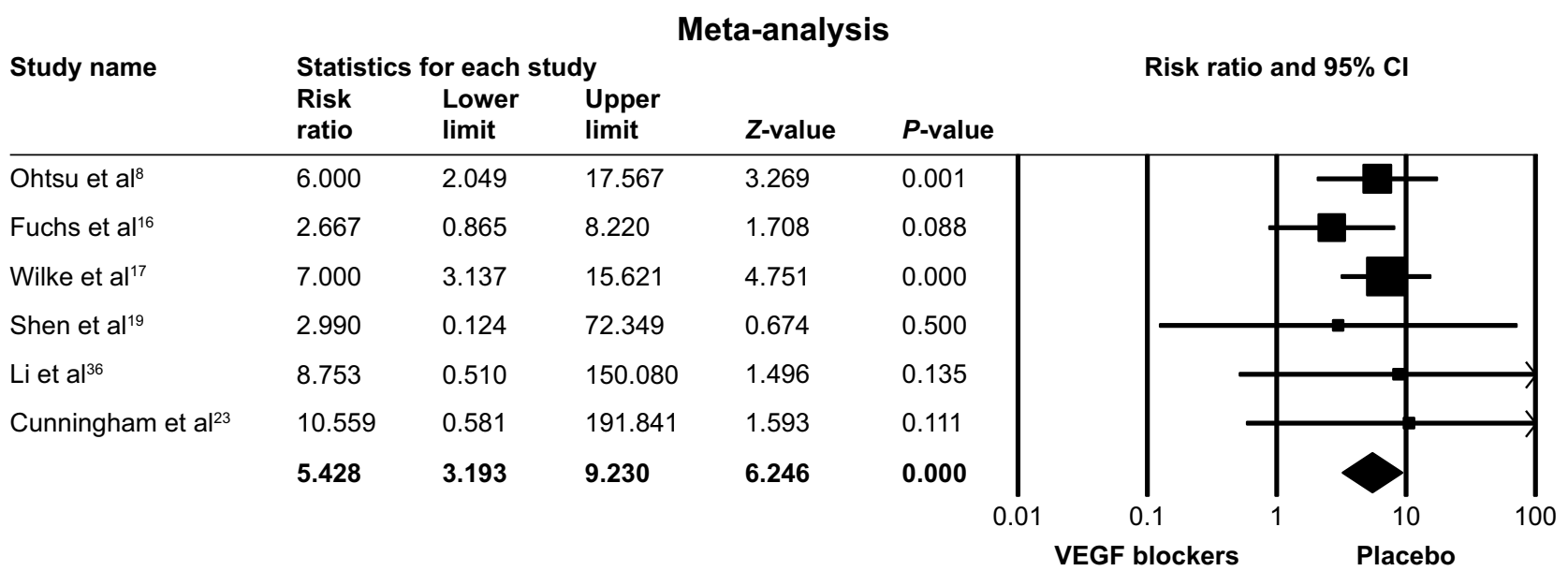

Figure 5 Forest plot for hypertension morbidity in patients with or without vascular endothelial growth factor (VEGF) or vascular endothelial growth factor receptor (VEGFR) blocker in addition to chemotherapy.

Notes: A fixed effect model was used due to non-significant heterogeneity of publications $\left(I^{2}<0.0 \mathrm{I}, \mathrm{P}=0.780\right)$. Effect size was assessed by risk ratio and $95 \% \mathrm{Cl}$, and the hypertension was in favor of placebo treatment in addition to chemotherapy (risk ratio: $5.428 ; 95 \% \mathrm{Cl}$ : 3.193-9.230; $P<0.00$ I). Bold values represent the median. 


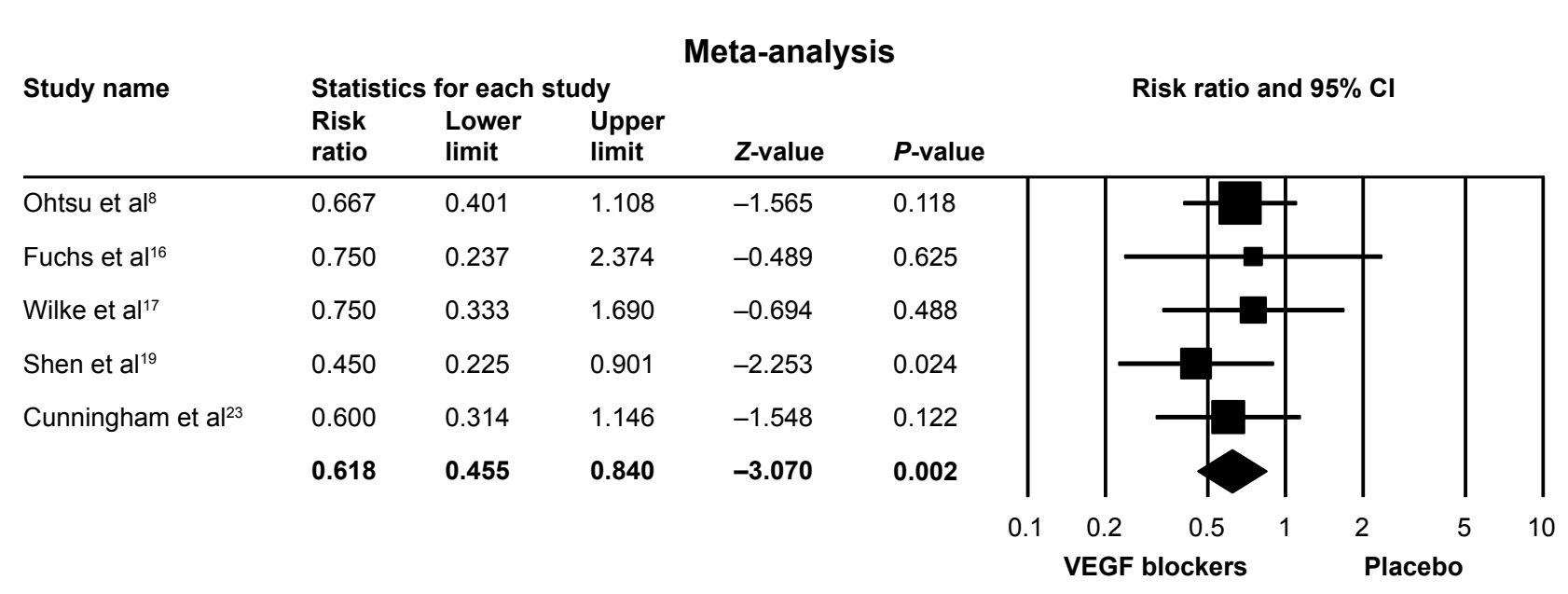

Figure 6 Forest plot for vomiting morbidity in patients with or without vascular endothelial growth factor (VEGF) or vascular endothelial growth factor receptor (VEGFR) blocker in addition to chemotherapy.

Notes: A fixed effect model was used due to non-significant heterogeneity of publications $\left(I^{2}<0.0 \mathrm{I}, P=0.8 \mathrm{I}\right)$. Effect size was assessed by risk ratio and $95 \% \mathrm{Cl}$, and the hypertension was in favor of VEGF or VEGFR blocker in addition to chemotherapy (risk ratio: $0.599 ; 95 \% \mathrm{Cl}$ : $0.43 \mathrm{I}-0.833 ; P=0.002$ ). Bold values represent the median.

VEGF/VEGFR blockers had higher risk in terms of hypertension morbidity, which was significantly different between the two groups (risk ratio: 5.428 ; $95 \%$ CI: $3.193-9.230$; $P<0.001)$. Interestingly, however, morbidity of vomiting was favorably less in patients with VEGF/VEGFR blockers in combination with chemotherapy than that in patients treated with placebo (risk ratio: 0.618 ; $95 \%$ CI: $0.455-0.840$; $P<0.001$, Figure 6). Similarly, risk of neutropenia morbidity was slightly but not significantly higher in the patients with VEGF/VEGFR blockers (risk ratio: 1.102; 95\% CI: $0.961-0.1 .265 ; P=0.164$, Figure 7 ), while risk of anemia was favorably less (risk ratio: 0.842 ; $95 \%$ CI: $0.644-1.102$; $P=0.210$, Figure 8 ) in the patients treated with VEGF or
VEGFR blockers although neither was significantly different between the two groups $(P>0.05)$.

\section{Risk of bias in individual studies}

As shown in Figure S1, Begg's funnel plot of standard error by $\log$ odds ratio indicated that there was no significant evidence for publication bias.

\section{Discussion}

Gastric cancer is the second leading cause of cancerrelated death. Despite recent progress in understanding the molecular biology of gastric cancer and the related signaling pathways offer promising treatment for selected groups of

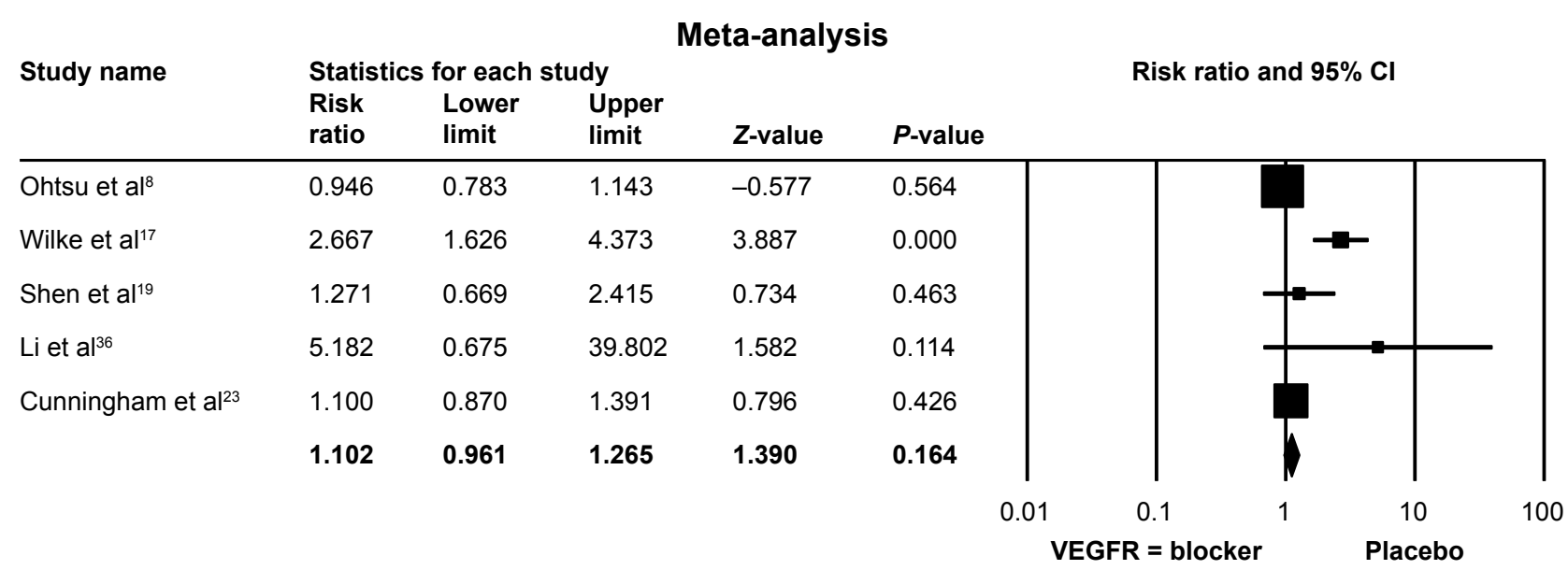

Figure 7 Forest plot for neutropenia morbidity in patients with or without vascular endothelial growth factor (VEGF) or vascular endothelial growth factor receptor (VEGFR) blocker in addition to chemotherapy.

Notes: $\mathrm{A}$ random effect model was used due to significant heterogeneity of publications $\left(I^{2}=75.46, P=0.003\right)$. Effect size was assessed by risk ratio and $95 \% \mathrm{Cl}$, and the hypertension was in favor of placebo treatment in addition to chemotherapy, which was without significant difference (risk ratio: I. I02; $95 \% \mathrm{Cl}$ : $0.96 \mathrm{I}-0.1 .265$; $P=0.164$ ). Bold values represent the median. 


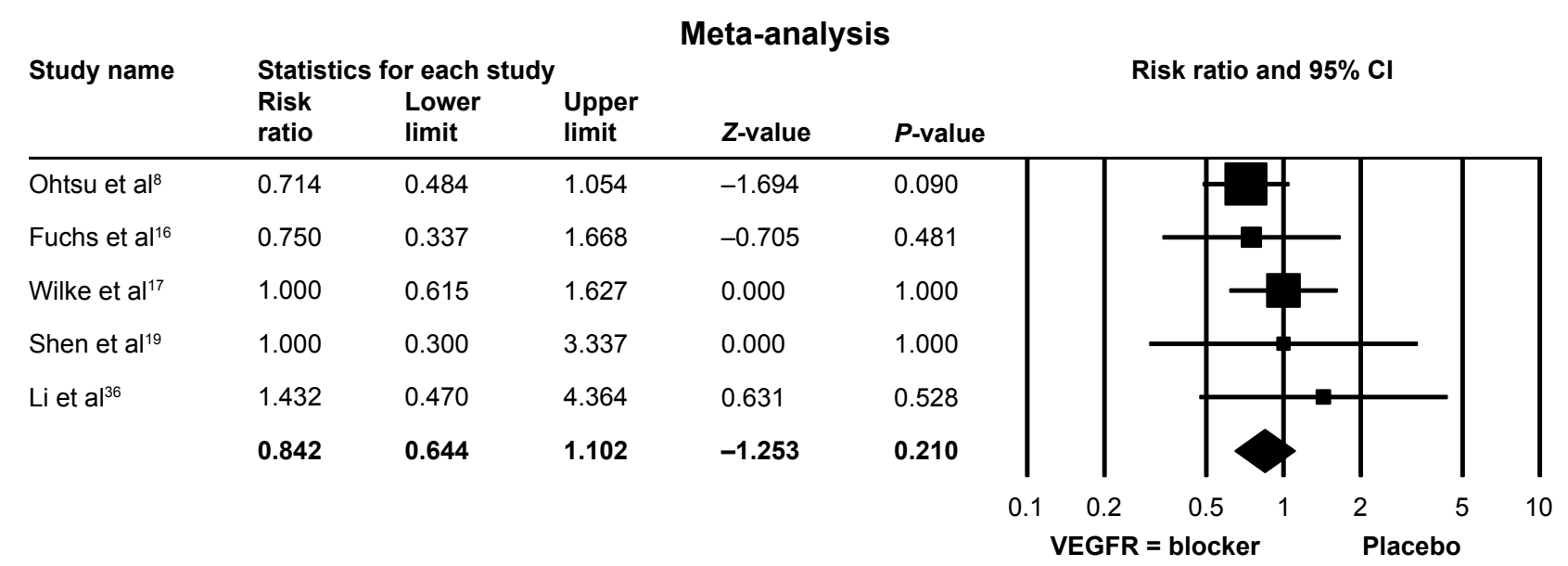

Figure 8 Forest plot for anemia morbidity in patients with or without vascular endothelial growth factor (VEGF) or vascular endothelial growth factor receptor (VEGFR) blocker in addition to chemotherapy.

Notes: A fixed effect model was used due to non-significant heterogeneity of publications $\left(I^{2}<0.0 \mathrm{I}, P=0.685\right)$. Effect size was assessed by risk ratio and $95 \% \mathrm{Cl}$, and the hypertension was in favor of VEGF or VEGFR blocker in addition to chemotherapy, which was without significant difference (risk ratio: $0.842 ; 95 \% \mathrm{Cl}: 0.644-1.102 ; P=0.210$ ). Bold values represent the median.

patients, OS and PFS rate are still poor. Recently, however, targeted therapies have significantly impacted the treatment strategy of several common solid tumor gastric cancer. In this regard, several antiangiogenesis reagents have been approved for the treatment of advanced or metastatic gastric cancer. Here, we systematically reviewed and performed meta-analysis on clinical trials for the treatment of advanced or metastatic gastric cancers with antiangiogenesis reagents including anti-VEGF mAb (bevacizumab), anti-VEGFR $\mathrm{mAb}$ (ramucirumab) and selective VEGFR TKI (apatinib). It was found that median OS time was significantly longer in the patients treated with VEGF/VEGFR blockers compared to that in the patients without the blockers when all of 8 selected Phase III clinical trials were analyzed together, although not all of the trials achieved positive results. ${ }^{8,19,23} \mathrm{In}$ addition, PFS time was also significantly longer in patients treated with VEGF/VEGFR blockers than that in patients treated with placebo. Interestingly, 4 out of the selected 8 Phase III trials tested bevacizumab and meta-analysis on these 4 studies indicated that there was no significant difference between bevacizumab and placebo with or without combination of other chemotherapeutic reagents. By contrast, 3 out of the selected 8 Phase III trials tested ramucirumab and meta-analysis on these 3 studies revealed that ramucirumab was significantly favored in the treatment of gastric cancer. In addition, patients treated with VEGF/ VEGFR blockers had higher morbidity of hypertension and neutropenia but had less risk side effects of vomiting and anemia. These findings suggest that addition of antiangiogenesis reagents to the first- or second-line chemotherapy, especially the anti-VEGFR-mAb, prolongs patients' OS and PFS time in advanced or metastatic gastric cancer.

The pathogenesis of gastric cancer involves multiple alteration of signaling pathways including epithermal growth factor (EGF) and its receptor (HER2), VEGF and its receptor (VEGFR). Novel mAbs selectively targeting these growth factors and their receptors have been developed for the treatment of gastric cancer. In this context, the addition of trastuzumab has significantly improved survival in patients with HER2-positive gastric cancer. ${ }^{39}$ However, this therapeutic option is available only for a few patients, as the overexpression or amplification of HER 2 has been identified in $<20 \%$ of patients. ${ }^{39,40}$

In contrast to EGF/EGFR signaling, studies have indicated that activation of VEGF/VEGFR signaling promotes aberrant tumor angiogenesis, which could be a potential target in variety of solid cancers including gastric cancer. To date, following strategies have been developed to target angiogenic signaling in solid tumors including gastric cancer: 1) targeting pro-angiogenic factor (VEGF) with $\mathrm{mAb}$ (bevacizumab); 2) targeting VEGF receptors (VEGFR) with $\mathrm{mAb}$ (ramucirumab) and 3) selectively targeting intracellular signaling with TKI (sunitinib, sorafenib, apatinib). In the current review, therefore, Phase I-III clinical trials on gastric cancer with anti-VEGF-mAb, anti-VEGFR-mAb and selective VEGFR downstream TKI were selected and systematically reviewed, and furthermore, meta-analysis on OS and PFS was performed.

While several tumors including gastric cancer are sensitive to VEGF inhibitors, no biomarkers of response to 
antiangiogenic agents were identified in clinical practice. However, since angiogenesis plays a key role in the development and progression of gastric cancer and increased expression of VEGF pathway proteins in most of human cancers, agents that specifically target angiogenesis in gastric cancer including anti-VEGF-mAb, anti-VEGFR-mAb and selective VEGFR downstream TKI are desirable.

Bevacizumab is a humanized mAb targeting VEGF-A, a protein playing a significant role in angiogenesis. This $\mathrm{mAb}$ had been tested in the AVAGAST Phase III trial. ${ }^{8}$ In this Phase III trial, 774 patients were enrolled and the combination of cisplatin and fluoropyrimidine with and without bevacizumab in the first-line treatment was compared. Unfortunately, this trial failed to meet the primary endpoint (OS). Consistent with result of this Phase III trial, metaanalysis of the current study in this clinical trial together with other 3 Phase III clinical trials ${ }^{18,23}$ indicated that addition of bevacizumab on top of the first- or second-line chemotherapy failed to prolong patients' life. In addition, a Phase Ib/II study, which tested weekly docetaxel and cisplatin together with capecitabine and bevacizumab for the treatment of advanced gastric cancer, was closed early because of the accumulation of toxicity-related deaths. ${ }^{12}$ These findings suggested that strategy of directly targeting pro-angiogenic factor (VEGF-A) with $\mathrm{mAb}$ is not a promising strategy.

Ramucirumab is a fully humanized $\mathrm{mAb}$ targeting VEGFR-2 and it has been approved by FDA as a single agent or in combination with fluoropyrimidine- or platinumcontaining chemotherapeutic agent for the treatment of patients with advanced or metastatic gastric cancer. VEGFR-2 is the main mediator of angiogenic signaling in endothelial cells and a primary responder to VEGF. VEGFR-2 activation plays a crucial role in tumor angiogenesis, and inhibition of the VEGFR-2 signaling pathway has become an attractive approach for cancer therapy. Most recently, a systematic review and meta-analysis indicated that VEGFR-2 overexpression is a promising negative prognosis predictor for patients with gastric cancer. ${ }^{41}$ The REGARD ${ }^{16}$ and RAINBOW ${ }^{17}$ randomized Phase III clinical trials tested the efficacy of ramucirumab in advanced/metastatic pretreated gastric cancers; the primary end point (OS rate) was met in both studies. In the REGARD trial, ${ }^{16}$ median OS was 5.2 vs 3.8 months and median PFS was 2.1 vs 1.3 months with advantage in the ramucirumab arm. In the RAINBOW study, ${ }^{17}$ median OS was 9.6 vs 7.4 months and median PFS was 4.4 vs 2.9 months with advantage in the ramucirumab group. Consistently, meta-analysis of the current study on the total of 3 out of the selected 8 Phase III clinical trials demonstrated that patients who received ramucirumab had significantly longer OS and PFS compared to the patients who received placebo, suggesting that targeting VEGF receptor is a promising strategy for the treatment of gastric cancer.

Similar to sunitinib and sorafenib, apatinib is a TKI that selectively blocks downstream signaling of VEGFR. Although 13 Phase I or II clinical trials on the VEGFR-TKI (sunitinib, sorafenib or apatinib) have been reported, ${ }^{24-36}$ only one Phase III trial on apatinib consisting of 267 patients was reported,,$^{22}$ and it was found that median OS and PF were significantly improved in the apatinib group $(6.5 \mathrm{~m}$ and $2.6 \mathrm{~m}$ ) compared with the placebo group $(4.7 \mathrm{~m}$ and $1.8 \mathrm{~m}$, respectively). However, adverse events such as hand-foot syndrome, proteinuria and hypertension were also higher in the patients who received apatinib. ${ }^{22}$ In addition to aforementioned TKIs, several Phase I and II studies have also tested antitumor effect of several types of TKIs including axitinib, ${ }^{11}$ pazopanib $^{20,42}$ and regorafenib. ${ }^{43,44}$ Of them, pazopanib and regorafenib are orally bioavailable and multitargeted TKI, mainly targeting VEGFR2, PDGFR and FGFR tyrosine kinase. ${ }^{42,45}$ It has been reported that pazopanib alone or the combination of pazopanib and capecitabine and oxaliplatin showed moderate antitumor activity and an acceptable toxicity when they were used as a first-line treatment in metastatic/recurrent advanced gastric cancer patients; ${ }^{46,47}$ and that regorafenib was effective in prolonging PFS in refractory advanced gastric adenocarcinoma. ${ }^{43}$ However, antitumor effect of these TKIs remains to be further determined through randomized and controlled clinical trials.

Preliminary pharmacokinetic data from patients enrolled into the selected 8 Phase III clinical trials found increased toxicity with higher exposures of bevacizumab and ramucirumab. In this regard, adverse effects including hypertension, neutropenia, vomiting, anemia and embolism have been reported. Interestingly, patients treated with VEGF or VEGFR mAbs had higher morbidity of hypertension and neutropenia, but less risk in the morbidity of vomiting and anemia. The mechanisms of these side effects, however, remain to be further investigated.

There are several limitations in the current study. First, limited number of Phase III clinical trials were enrolled into this study, which remains to be further updated with findings of accumulated clinical trials. Second, due to limited number of the randomized clinical trials, sub-group analysis on the gastric cancers in terms of tumor stages, metastasis status, location of the cancer in the gastroenterological tract and ethnic difference was not performed. In this regard, it has been reported that $V E G F-634 \mathrm{G}>\mathrm{CC}$ allele and $\mathrm{GG}$ genotype 
were associated with gastric cancer risk in Caucasians, while $V E G F+1612 \mathrm{G} /$ A gen polymorphism was associated with gastric cancer risk for the Asian population, ${ }^{41}$ and that ethnic difference was associated with outcomes of TKI treatment between Caucasian and Asian patients with malignant tumor. ${ }^{48}$ Third, only one Phase III trial on the VEGF receptor TKI (apatinib) was enrolled and analyzed together with other studies with anti-VEGF-A mAb or anti-VEGFR mAb, and thus more randomized clinical trials data on VEGFR-TKI are necessary to confirm the benefit of selective VEGFR-TKI on gastric cancer treatment.

Taken together, in the current study, general therapeutic and side effects of anti-VEGF mAb, anti-VEGFR mAb and anti-VEGFR-TKI with or without other chemotherapeutic drugs for the treatment of gastric cancer were systematically reviewed followed by meta-analysis performance. Compared with the placebo, antiangiogenic reagents with or without combination of chemotherapeutic drugs could significantly prolong gastric cancer patients' median OS and PFS. Furthermore, treatment with the anti-VEGFR $\mathrm{mAb}$ ramucirumab, but not the anti-VEGF mAb bevacizumab, resulted in significant improvement of median OS and PFS. In addition, patients who received anti-VEGF-mAb or antiVEGFR-mAb had higher morbidity of hypertension and neutropenia, but less risk in the morbidity of vomiting and anemia. These findings suggested that a fully humanized anti-VEGFR mAb could be an effective therapeutic agent for the treatment of gastric cancer.

\section{Acknowledgment}

This work was supported by the Beijing Municipal Administration of Hospitals Clinical Medicine Development of Special Funding (ZYLX201504), National Key Technologies R\&D Program (No 2015BAI13B09) and Support Project of High-level Teachers in Beijing Municipal Universities in the Period of 13th Five-year Plan (IDHT20170516).

\section{Disclosure}

The authors report no conflicts of interest in this work.

\section{References}

1. Ferrara N, Gerber HP, LeCouter J. The biology of VEGF and its receptors. Nat Med. 2003;9(6):669-676.

2. Alitalo K, Carmeliet P. Molecular mechanisms of lymphangiogenesis in health and disease. Cancer Cell. 2002;1(3):219-227.

3. Shalaby F, Rossant J, Yamaguchi TP, et al. Failure of blood-island formation and vasculogenesis in Flk-1-deficient mice. Nature. 1995; 376(6535):62-66.

4. Kim M, Park HJ, Seol JW, et al. VEGF-A regulated by progesterone governs uterine angiogenesis and vascular remodelling during pregnancy. EMBO Mol Med. 2013;5(9):1415-1430.
5. Higgins J, Thompson S, Deeks J, Altman D. Statistical heterogeneity in systematic reviews of clinical trials: a critical appraisal of guidelines and practice. J Health Serv Res Policy. 2002;7(1):51-61.

6. Thompson SG, Higgins JP. How should meta-regression analyses be undertaken and interpreted? Stat Med. 2002;21(11):1559-1573.

7. El-Rayes BF, Zalupski M, Bekai-Saab T, et al. A phase II study of bevacizumab, oxaliplatin, and docetaxel in locally advanced and metastatic gastric and gastroesophageal junction cancers. Ann Oncol. 2010;21(10):1999-2004.

8. Ohtsu A, Shah MA, Van Cutsem E, et al. Bevacizumab in combination with chemotherapy as first-line therapy in advanced gastric cancer: a randomized, double-blind, placebo-controlled phase III study. J Clin Oncol. 2011;29(30):3968-3976.

9. Masuzawa T, Fujiwara Y, Okada K, et al. Phase I/II study of S-1 plus cisplatin combined with peptide vaccines for human vascular endothelial growth factor receptor 1 and 2 in patients with advanced gastric cancer. Int J Oncol. 2012;41(4):1297-1304.

10. Uronis HE, Bendell JC, Altomare I, et al. A phase II study of capecitabine, oxaliplatin, and bevacizumab in the treatment of metastatic esophagogastric adenocarcinomas. Oncologist. 2013;18(3):271-272.

11. Ueda S, Satoh T, Gotoh M, Gao L, Doi T. A phase ib study of safety and pharmacokinetics of ramucirumab in combination with paclitaxel in patients with advanced gastric adenocarcinomas. Oncologist. 2015; 20(5):493-494.

12. Brenner B, Sarfaty M, Purim O, et al. A phase Ib/II study evaluating the combination of weekly docetaxel and cisplatin together with capecitabine and bevacizumab in patients with advanced esophago-gastric cancer. PLoS One. 2016;11(7):e0157548.

13. Ku GY, Bains MS, Park DJ, et al. Phase II study of bevacizumab and preoperative chemoradiation for esophageal adenocarcinoma. J Gastrointest Oncol. 2016;7(6):828-837.

14. Meulendijks D, Beerepoot LV, Boot H, et al. Trastuzumab and bevacizumab combined with docetaxel, oxaliplatin and capecitabine as firstline treatment of advanced HER2-positive gastric cancer: a multicenter phase II study. Invest New Drugs. 2016;34(1):119-128.

15. Kim JH, Park SR, Ryu MH, et al. Phase II study of induction chemotherapy with docetaxel, capecitabine, and cisplatin plus bevacizumab for initially unresectable gastric cancer with invasion of adjacent organs or paraaortic lymph node metastasis. Cancer Res Treat. 2018; 50(2):518-529.

16. Fuchs CS, Tomasek J, Yong CJ, et al. Ramucirumab monotherapy for previously treated advanced gastric or gastro-oesophageal junction adenocarcinoma (REGARD): an international, randomised, multicentre, placebo-controlled, phase 3 trial. Lancet. 2014;383(9911):31-39.

17. Wilke H, Muro K, Van Cutsem E, et al; RAINBOW Study Group. Ramucirumab plus paclitaxel versus placebo plus paclitaxel in patients with previously treated advanced gastric or gastro-oesophageal junction adenocarcinoma (RAINBOW): a double-blind, randomised phase 3 trial. Lancet Oncol. 2014;15(11):1224-1235.

18. Ma J, Yao S, Li XS, Kang HR, Yao FF, Du N. Neoadjuvant therapy of DOF regimen plus bevacizumab can increase surgical resection ratein locally advanced gastric cancer: a randomized, controlled study. Medicine (Baltimore). 2015;94(42):e1489.

19. Shen $\mathrm{L}, \mathrm{Li} J, \mathrm{Xu} J$, et al. Bevacizumab plus capecitabine and cisplatin in Chinese patients with inoperable locally advanced or metastatic gastric or gastroesophageal junction cancer: randomized, double-blind, phase III study (AVATAR study). Gastric Cancer. 2015;18(1):168-176.

20. Al-Batran SE, Van Cutsem E, Oh SC, et al. Quality-of-life and performance status results from the phase III RAINBOW study of ramucirumab plus paclitaxel versus placebo plus paclitaxel in patients with previously treated gastric or gastroesophageal junction adenocarcinoma. Ann Oncol. 2016;27(4):673-679.

21. Chen H, Zhang J, Cao G, et al. Target hepatic artery regional chemotherapy and bevacizumab perfusion in liver metastatic colorectal cancer after failure of first-line or second-line systemic chemotherapy. Anticancer Drugs. 2016;27(2):118-126. 
22. Brambilla E, Le Teuff G, Marguet S, et al. Prognostic effect of tumor lymphocytic infiltration in resectable non-small-cell lung cancer. J Clin Oncol. 2016;34(11):1223-1230.

23. Cunningham D, Stenning SP, Smyth EC, et al. Peri-operative chemotherapy with or without bevacizumab in operable oesophagogastric adenocarcinoma (UK Medical Research Council ST03): primary analysis results of a multicentre, open-label, randomised phase 2-3 trial. Lancet Oncol. 2017;18(3):357-370.

24. Bang YJ, Kang YK, Kang WK, et al. Phase II study of sunitinib as second-line treatment for advanced gastric cancer. Invest New Drugs. 2011;29(6):1449-1458.

25. Boku N, Muro K, Machida N, et al. Phase I study of sunitinib plus S-1 and cisplatin in Japanese patients with advanced or metastatic gastric cancer. Invest New Drugs. 2014;32(2):261-270.

26. Yi JH, Lee J, Lee J, et al. Randomised phase II trial of docetaxel and sunitinib in patients with metastatic gastric cancer who were previously treated with fluoropyrimidine and platinum. Br J Cancer. 2012;106(9):1469-1474.

27. Moehler M, Gepfner-Tuma I, Maderer A, et al. Sunitinib added to FOLFIRI versus FOLFIRI in patients with chemorefractory advanced adenocarcinoma of the stomach or lower esophagus: a randomized, placebo-controlled phase II AIO trial with serum biomarker program. BMC Cancer. 2016;16:699.

28. Janjigian YY, Vakiani E, Ku GY, et al. Phase II trial of sorafenib in patients with chemotherapy refractory metastatic esophageal and gastroesophageal (GE) junction cancer. PLoS One. 2015;10(8): e0134731

29. Sun W, Powell M, O’Dwyer PJ, Catalano P, Ansari RH, Benson AB 3rd. Phase II study of sorafenib in combination with docetaxel and cisplatin in the treatment of metastatic or advanced gastric and gastroesophageal junction adenocarcinoma: ECOG 5203. J Clin Oncol. 2010;28(18):2947-2951.

30. Lee KW, Park SR, Oh DY, et al. Phase I study of sunitinib plus capecitabine/cisplatin or capecitabine/oxaliplatin in advanced gastric cancer. Invest New Drugs. 2013;31(6):1547-1558.

31. Gómez-Martin C, Salazar R, Montagut C, et al. A phase I, dosefinding study of sunitinib combined with cisplatin and 5-fluorouracil in patients with advanced gastric cancer. Invest New Drugs. 2013;31(2): 390-398.

32. Moehler M, Mueller A, Hartmann JT, et al. An open-label, multicentre biomarker-oriented AIO phase II trial of sunitinib for patients with chemo-refractory advanced gastric cancer. Eur J Cancer. 2011;47(10): 1511-1520.

33. Martin-Richard M, Gallego R, Pericay C, et al. Multicenter phase II study of oxaliplatin and sorafenib in advanced gastric adenocarcinoma after failure of cisplatin and fluoropyrimidine treatment. A GEMCAD study. Invest New Drugs. 2013;31(6):1573-1579.

34. Yamada Y, Kiyota N, Fuse N, et al. A phase I study of sorafenib in combination with $\mathrm{S}-1$ plus cisplatin in patients with advanced gastric cancer. Gastric Cancer. 2014;17(1):161-172.
35. Kim C, Lee JL, Choi YH, et al. Phase I dose-finding study of sorafenib in combination with capecitabine and cisplatin as a first-line treatment in patients with advanced gastric cancer. Invest New Drugs. 2012;30(1): 306-315.

36. Li J, Qin S, Xu J, et al. Apatinib for chemotherapy-refractory advanced metastatic gastric cancer: results from a randomized, placebo-controlled, parallel-arm, phase II trial. J Clin Oncol. 2013;31(26):3219-3225.

37. Haas NB, Manola J, Uzzo RG, et al. Adjuvant sunitinib or sorafenib for high-risk, non-metastatic renal-cell carcinoma (ECOG-ACRIN E2805): a double-blind, placebo-controlled, randomised, phase 3 trial. Lancet. 2016;387(10032):2008-2016

38. Shah MA, Jhawer M, Ilson DH, et al. Phase II study of modified docetaxel, cisplatin, and fluorouracil with bevacizumab in patients with metastatic gastroesophageal adenocarcinoma. J Clin Oncol. 2011;29(7):868-874.

39. Bang YJ, Van Cutsem E, Feyereislova A, et al. Trastuzumab in combination with chemotherapy versus chemotherapy alone for treatment of HER2-positive advanced gastric or gastro-oesophageal junction cancer (ToGA): a phase 3, open-label, randomised controlled trial. Lancet. 2010;376(9742):687-697.

40. Janjigian YY, Werner D, Pauligk C, et al. Prognosis of metastatic gastric and gastroesophageal junction cancer by HER2 status: a European and USA International collaborative analysis. Ann Oncol. 2012;23(10):2656-2662.

41. Liu W, Dong Z, Hu R, Wang C. Association of vascular endothelial growth factor ( VEGF) gene polymorphisms with gastric cancer and its development, prognosis, and survival. Technol Cancer Res Treat. 2018; $17: 1533034617753810$.

42. Kim ST, Jang HL, Lee SJ, et al. Pazopanib, a novel multitargeted kinase inhibitor, shows potent in vitro antitumor activity in gastric cancer cell lines with FGFR2 amplification. Mol Cancer Ther. 2014; 13(11):2527-2536.

43. Pavlakis N, Sjoquist KM, Martin AJ, et al. Regorafenib for the treatment of advanced gastric cancer (INTEGRATE): a multinational placebocontrolled Phase II trial. J Clin Oncol. 2016;34(23):2728-2735.

44. Smyth EC. Regorafenib in gastric cancer. Transl Gastroenterol Hepatol. 2017;2:16.

45. Cha Y, Kim HP, Lim Y, Han SW, Song SH, Kim TY. FGFR2 amplification is predictive of sensitivity to regorafenib in gastric and colorectal cancers in vitro. Mol Oncol. 2018;12(7):993-1003.

46. Ahn HK, Choi JY, Kim KM, et al. Phase II study of pazopanib monotherapy in metastatic gastroenteropancreatic neuroendocrine tumours. Br J Cancer. 2013;109(6):1414-1419.

47. Kim ST, Ahn S, Lee J, et al. Value of FGFR2 expression for advanced gastric cancer patients receiving pazopanib plus CapeOX (capecitabine and oxaliplatin). J Cancer Res Clin Oncol. 2016;142(6):1231-1237.

48. Liu X, Fiocco M, Swen JJ, Guchelaar HJ. Assessment of ethnic differences in sunitinib outcome between Caucasian and Asian patients with metastatic renal cell carcinoma: a meta-analysis. Acta Oncol. 2017; 56(4):582-589. 


\section{Supplementary material}

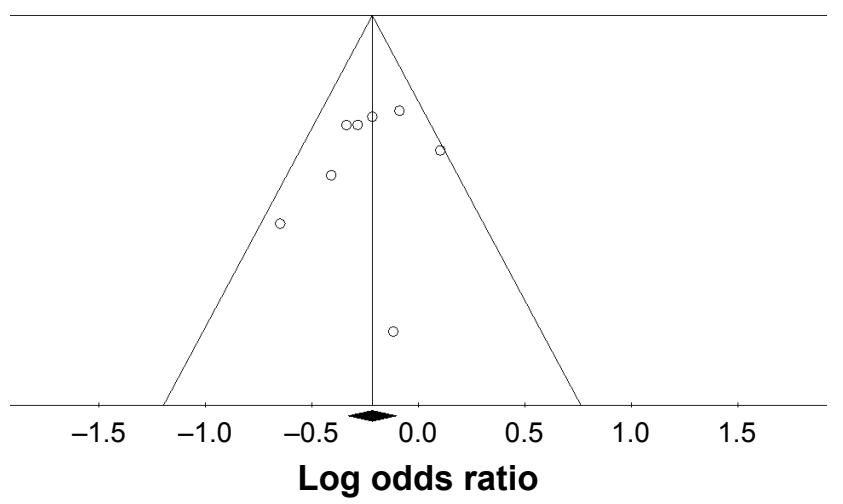

Figure SI Funnel plot of standard error by log odds ratio assessing publication bias.

\section{Publish your work in this journal}

OncoTargets and Therapy is an international, peer-reviewed, open access journal focusing on the pathological basis of all cancers, potential targets for therapy and treatment protocols employed to improve the management of cancer patients. The journal also focuses on the impact of management programs and new therapeutic agents and protocols on patient perspectives such as quality of life, adherence and satisfaction. The manuscript management system is completely online and includes a very quick and fair peer-review system, which is all easy to use. Visit http://www.dovepress.com/testimonials.php to read real quotes from published authors.

\footnotetext{
Submit your manuscript here: http://www.dovepress.com/oncotargets-and-therapy-journal
} 